\title{
TRIB3 R84 variant affects glucose homeostasis by altering the interplay between insulin sensitivity and secretion
}

\author{
S. Prudente $\cdot$ R. Baratta $~ F$. Andreozzi $\cdot$ E. Morini $\cdot$ M. G. Farina $\cdot$ A. Nigro $\cdot$ \\ M. Copetti - F. Pellegrini • E. Succurro • L. Di Pietrantonio • C. Brufani • F. Barbetti • \\ B. Dallapiccola $\cdot$ G. Sesti $・$ V. Trischitta $\cdot$ L. Frittitta
}

Received: 23 December 2009 / Accepted: 4 March 2010/Published online: 15 April 2010

(C) Springer-Verlag 2010

\footnotetext{
V. Trischitta and L. Frittitta contributed equally to this study.

S. Prudente $\cdot$ E. Morini $\cdot$ L. Di Pietrantonio $\cdot$ B. Dallapiccola $•$ V. Trischitta $(\triangle)$

IRCCS Casa Sollievo della Sofferenza Mendel Institute,

Viale Regina Margherita 261,

00198 Rome, Italy

e-mail: vincenzo.trischitta@uniroma1.it

R. Baratta $\cdot$ M. G. Farina $\cdot$ A. Nigro $\cdot$ L. Frittitta $(\bowtie)$

Unit of Endocrinology, Department of Internal and Specialist

Medicine, University of Catania Medical School,

Garibaldi Hospital,

Via Palermo 636,

I-95122 Catania, Italy

e-mail: 1fritti@unict.it
}

F. Andreozzi $\cdot$ E. Succurro $\cdot$ G. Sesti

Department of Experimental and Clinical Medicine,

University Magna Græcia,

Catanzaro, Italy

\begin{abstract}
Aims/hypothesis The results of studies on the genetics of
complex traits need to be replicated and to reach robust
statistical significance before they can be considered as
established. We here tried to replicate the previously
reported association between the TRIB3 Q84R polymor-
Abstract
Aims/hypothesis The results of studies on the genetics of
complex traits need to be replicated and to reach robust
statistical significance before they can be considered as
established. We here tried to replicate the previously
reported association between the TRIB3 Q84R polymor-

Abstract
Aims/hypothesis The results of studies on the genetics of
complex traits need to be replicated and to reach robust
statistical significance before they can be considered as
established. We here tried to replicate the previously
reported association between the TRIB3 Q84R polymor-

Abstract
Aims/hypothesis The results of studies on the genetics of
complex traits need to be replicated and to reach robust
statistical significance before they can be considered as
established. We here tried to replicate the previously
reported association between the TRIB3 Q84R polymor-

Abstract
Aims/hypothesis The results of studies on the genetics of
complex traits need to be replicated and to reach robust
statistical significance before they can be considered as
established. We here tried to replicate the previously
reported association between the TRIB3 Q84R polymor-

Abstract
Aims/hypothesis The results of studies on the genetics of
complex traits need to be replicated and to reach robust
statistical significance before they can be considered as
established. We here tried to replicate the previously
reported association between the TRIB3 Q84R polymorphism (rs2295490) and glucose homeostasis.

Methods Three samples of Europeans with fasting glucose $<7.0 \mathrm{mmol} / 1$ were studied. In sample $1(n=791)$, the association between TRIB3 Q84R and impaired glucose regulation (IGR; defined as impaired fasting glucose and/or impaired glucose tolerance and/or type 2 diabetes by OGTT) and insulin sensitivity (ISI), and its interplay with early-phase insulin secretion (i.e. disposition index [DI]) were analysed. Sample $2(n=374)$ and sample $3(n=394)$
\end{abstract}

were used to replicate the association with IGR and insulin sensitivity (by glucose clamp), respectively. Genotyping was performed by TaqMan allele discrimination.

Results R84 carriers were at higher risk of IGR: OR for the additive model 1.54, $p=0.004$, and 1.63, $p=0.027$, in samples 1 and 2 , respectively. In sample 1 , both ISI $(p=0.005)$ and DI $(p=0.043)$ were progressively lower from QQ to $\mathrm{QR}$ and $\mathrm{RR}$ individuals. A 'triangulation approach' indicated that the association with IGR was mostly mediated by DI rather than by ISI changes (i.e. being the expected ORs 1.51 and 1.25, respectively). In sample 3, glucose disposal was $38.8 \pm 17.7$, $33.8 \pm 14.4$, and $31.6 \pm 13.3 \mu \mathrm{mol} \mathrm{min} \mathrm{kg}^{-1}, p=0.022$, in QQ, QR and RR individuals, respectively.

Conclusions/interpretation Our data confirm that the TRIB3 R84 variant affects glucose homeostasis and suggest this

\author{
M. Copetti $\cdot$ F. Pellegrini \\ Unit of Biostatistics, IRCCS Casa Sollievo della Sofferenza, \\ San Giovanni Rotondo, Italy \\ C. Brufani $\cdot$ F. Barbetti \\ IRCCS Bambino Gesù Pediatric Hospital, \\ Rome, Italy \\ V. Trischitta \\ Research Unit of Diabetes and Endocrine Diseases, \\ IRCCS Casa Sollievo della Sofferenza, \\ San Giovanni Rotondo, Italy \\ V. Trischitta \\ Department of Medical Pathophysiology, 'Sapienza' University, \\ Rome, Italy
}

Present Address:

B. Dallapiccola

IRCCS Bambino Gesù Pediatric Hospital,

Rome, Italy 
effect is due to an alteration of the interplay between insulin sensitivity and secretion.

Keywords Akt Early type 2 diabetes .

Inhibitors of insulin signalling

$\begin{array}{ll}\text { Abbreviations } \\ \text { ANCOVA } & \text { Analysis of covariance } \\ \text { DI } & \text { Disposition index } \\ \text { HOMA-IR } & \text { HOMA of insulin resistance } \\ \text { IFG } & \text { Impaired fasting glucose } \\ \text { IGI } & \text { Insulinogenic index } \\ \text { IGR } & \text { Impaired glucose regulation } \\ \text { IGT } & \text { Impaired glucose tolerance } \\ \text { ISI } & \text { Insulin sensitivity index } \\ \text { NG } & \text { Normoglycaemic } \\ \text { SNP } & \text { Single nucleotide polymorphism }\end{array}$

\section{Introduction}

In insulin-resistant individuals who eventually develop type 2 diabetes, normal or near-normal glycaemia is maintained for many years by compensatory hyperinsulinaemia [1,2]. Hyperglycaemia eventually ensues when beta cells fail to secrete sufficient insulin to adequately counteract insulin resistance [3, 4]. Thus, an altered interplay between insulin sensitivity and secretion is instrumental for the development of abnormal glucose homeostasis [3, 4].

The mammalian tribbles homologue 3 (TRIB3) is an insulin signalling inhibitor which binds Akt [5] and inhibits [5-7] insulin-stimulated Akt phosphorylation and subsequent insulin action.

We have recently described a TRIB3 missense single nucleotide polymorphism (SNP) Q84R (i.e. rs2295490, where arginine replaces glutamine at position 84), resulting in a gain-of-function variant $[6,7]$. In vitro studies have revealed that the $\mathrm{R} 84$ variant is more effective than the Q84 variant in engaging Akt and reducing insulin-mediated Ser473 Akt phosphorylation in human insulin target tissues $[6,7]$ as well as in insulin-secreting beta cells (C. Wee Liew, J. Bochenski, J. Hu, A.S. Krowleski and R.N. Kulkarni, unpublished data), thus suggesting a deleterious role of this variant on both insulin action and secretion. In vivo, the R84 variant has been associated with insulin resistance $[6,8]$ and with type 2 diabetes (C. Wee Liew, J. Bochenski, J. Hu, A.S. Krowleski and R.N. Kulkarni, unpublished data); this latter association is likely to be secondary to an altered interplay between insulin secretion and insulin action observed in R84 carriers [9]. As for any genetic study of complex traits, these findings - despite coherence with the biological function of the Q84R polymorphism - need to be replicated as much as possible, with results reaching a high level of statistical significance before they can be considered established. Unfortunately, neither the Q84R polymorphism, nor any other SNP in good linkage disequilibrium with it, is included in the arrays utilised in the publicly available genome-wide association studies for type 2 diabetes and related traits $[10,11]$, thus precluding the possibility of performing additional in silico analyses and, conversely, indicating the need for further studies to address this issue.

Thus, in order to accumulate additional data on the association of the TRIB3 R84 variant with abnormal glucose homeostasis we studied several samples of Italians of European ancestry. In addition, we used a 'triangulation approach' [12] to get deeper insights into the mechanisms underlying this association.

\section{Methods}

Study participants and design

Three different samples of adult ( $\geq 18$ years of age) unrelated Italians of European ancestry with fasting plasma glucose $<7.0 \mathrm{mmol} / \mathrm{l}$ were studied.

Sample 1 comprised 791 unrelated adults (age 1872 years) from Eastern Sicily, with fasting plasma glucose $<7.0 \mathrm{mmol} / \mathrm{l}$, not taking medications known to interfere with glucose and lipid metabolism, who were recruited at the Endocrine Unit of Garibaldi Hospital (Catania, Italy). Of these, 196 were non-obese individuals (BMI $<30 \mathrm{~kg} / \mathrm{m}^{2}$, 80 men and 116 women, age $36.1 \pm 12.4$ years) recruited among the hospital staff and 595 were obese individuals (BMI $\geq 30 \mathrm{~kg} / \mathrm{m}^{2}, 158 \mathrm{men}$ and 437 women, age $35.5 \pm$ 11.7 years) recruited from the outpatient Obesity Clinic of the same institution. According to the American Diabetes Association 2003 criteria, 465 individuals (58.8\%) were normoglycaemic (NG: normal glucose levels both fasting and after OGTT), 326 (41.2\%) had impaired glucose regulation (IGR), including impaired fasting glucose (IFG, $n=102$ ) and/or impaired glucose tolerance (IGT, $n=177$ ) or type 2 diabetes $(n=47)$ as indicated by OGTT. No gene-byobesity status (i.e. non-obese or obese subgroup) interaction was observed in determining indices derived by OGTT data, including the insulin sensitivity index (ISI, see below) ( $p$ value for interaction $=0.49)$ and the disposition index (DI, see below) (adjusted $p=0.14$ ); thus, in order to increase the study statistical power, the two subgroups were pooled and analysed together after adjusting for age, sex and BMI. This sample was analysed for the association of the R84 variant with both glucose homeostasis and the interplay between insulin sensitivity and secretion as indicated by the DI obtained from the OGTT-derived data. 
Sample 2 comprised 374 unrelated obese (i.e. BMI $\geq$ $30 \mathrm{~kg} / \mathrm{m}^{2}$ ) patients (109 men and 265 women, age $41.3 \pm$ 13.6 years and BMI $42.3 \pm 8.4 \mathrm{~kg} / \mathrm{m}^{2}$ ) with fasting plasma glucose $<7.0 \mathrm{mmol} / \mathrm{l}$, consecutively recruited from the outpatient Obesity Clinic at the Department of Clinical Sciences of 'Sapienza' University in Rome. Of these, 141 individuals $(37.7 \%$ ) had IGR (either IFG, $n=29$, and/or IGT, $n=96$, or type 2 diabetes, $n=16$, as indicated by OGTT). This sample was analysed to try to replicate the association observed in sample 1 between the R84 variant and IGR.

Sample 3 comprised 394 individuals (153 men and 241 women, age $38.9 \pm 10.5$ years, BMI $29.3 \pm 6.7 \mathrm{~kg} / \mathrm{m}^{2}$ ) with fasting plasma glucose $<7 \mathrm{mmol} / \mathrm{l}$, consecutively recruited at the Department of Experimental and Clinical Medicine of the University 'Magna Graecia' of Catanzaro. Among these, 301 were offspring of patients with type 2 diabetes, with 178 having been previously analysed for the association between insulin sensitivity (as measured by the euglycaemichyperinsulinaemic glucose clamp technique [13]) and TRIB3 Q84R polymorphism [6]. This sample group was analysed to try to replicate the association observed in sample 1 between the R84 variant and insulin resistance.

All participants underwent physical examination including the measurements of height and weight and were kept on a weight-maintaining diet in the 4 days preceding the study. In sample 1, glucose, insulin, triacylglycerol levels and HDLcholesterol were measured using commercially available kits in blood specimens obtained after an overnight fast and immediately frozen at $-20^{\circ} \mathrm{C}$. The HOMA of insulin resistance (HOMA-IR) index was calculated as previously described [14]. An OGTT measuring baseline and $120 \mathrm{~min}$ glucose values was carried out for sample group 1; glucose and insulin values were also measured before and 30, 60, 90 and $120 \mathrm{~min}$ after glucose $(75 \mathrm{~g})$ load.

The study protocol was approved by the institutional review boards and performed according to the Helsinki Declaration. Written informed consent was obtained from each participant in the study.

\section{OGTT-derived measurements}

The disposition index is the product of the insulinogenic index (IGI) (as calculated according to the formula: [insulin $\{\mathrm{pmol} / \mathrm{l}\}$ at $30 \mathrm{~min}$-fasting plasma insulin $\{\mathrm{pmol} / \mathrm{l}\}] /$ [glucose $\{\mathrm{mmol} / \mathrm{l}\}$ at $30 \mathrm{~min}$-fasting plasma glucose $\{\mathrm{mmol} / \mathrm{l}\}]$ ) by the insulin sensitivity index, ISI (as calculated by the following formula: $10,000 / \sqrt{ }$ [fasting plasma glucose $\{\mathrm{mmol} / \mathrm{l}\} \times$ fasting plasma insulin $\{\mathrm{pmol} / \mathrm{l}\}] \times[$ mean OGTT glucose concentration $\{\mathrm{mmol} / 1\} \times$ mean OGTT insulin concentration $\{\mathrm{pmol} / 1\}])$. Of note, potential violations of the hyperbolic shape $(I G I \times I S I=k$, where $k$ is a constant value) were excluded through a goodness-of-fit test for the following non-linear model IGI $=k / I S I \quad\left(R^{2}=0.50\right.$, $p<0.0001)$.

Euglycaemic-hyperinsulinaemic glucose clamp

Euglycaemic-hyperinsulinaemic clamp was performed as previously described [13]. Briefly, after a $12 \mathrm{~h}$ overnight fast, a continuous insulin infusion was initiated at the rate of $40 \mathrm{mU} / \mathrm{m}^{2}$ of body surface area per min, after a priming dose, in order to reach and maintain a steady-state plasma insulin of about $625 \mathrm{pmol} / 1$. Plasma glucose was assessed at 5 min intervals during the $2 \mathrm{~h}$ clamp study by a glucose analyser. In the study participants, mean plasma glucose concentration during the last hour of the clamp was $5.16 \pm$ $0.3 \mathrm{mmol} / \mathrm{l}$. The mean amount of exogenous glucose infused during the last hour of the clamp period was used to calculate the rates of glucose disposal rate, and is

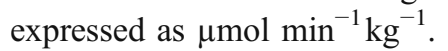

\section{Genotyping}

DNA was extracted from whole blood using a standard method. Genotyping was performed by TaqMan allele discrimination (assay C_16190162_10, Applied Biosystems, Forster City, CA, USA) on the HT7900 platform. The failure rate was $<1 \%$. Genotyping quality was checked by directly sequencing $10 \%$ of randomly selected samples. The agreement rate of re-sequenced samples was $100 \%$. The proportion of the Q84R genotypes obeyed HardyWeinberg equilibrium in all samples studied.

\section{Statistical analysis}

The general features of the study participants were expressed as means $\pm \mathrm{SD}$. The additive genetic model was a priori decided to be tested in all analyses. Because of the skewed distribution, fasting insulin, HOMA-IR and triacylglycerol values as well as ISI, IGI and DI indices, were log transformed before analysis.

Continuous variables were compared between groups using one-way or repeated-measurements analysis of covariance (ANCOVA). In particular, for glucose and insulin profiles during OGTT, repeated-measurements ANCOVA models to assess differences over time were carried out via hierarchical linear models; within-patient correlation was accounted for with an unstructured correlation-type matrix [15]. The exact test for Hardy-Weinberg equilibrium was carried out as previously described [16]. Multivariate logistic regression analysis was used to model the effect of the polymorphism on dichotomous outcomes and results were estimated as odd ratios with $95 \%$ confidence intervals.

An individual patient's data meta-analysis using a linear mixed model [17-19] was performed for abnormalities of 
glucose homeostasis (i.e. either type 2 diabetes or IGR, considered as a categorical trait) and for DI, considered as a continuous trait. Linear mixed models took into account potential between-sample heterogeneity (tested as a genotype-by-sample interaction). An unstructured correlation matrix was assumed. The results of categorical outcomes meta-analyses were reported as odds ratios with $95 \%$ confidence intervals, while the results of DI meta-analyses were reported as $\beta$ and $p$ values.

The possible presence of publication bias was visually assessed by funnel plots and statistically assessed by Begg and Mazumdar rank correlation test [20] and Egger's regression intercept test [21].

A 'triangulation approach' [12] was followed to determine whether association between polymorphisms of TRIB3 and IGR might be mediated by DI and ISI. The effect size per minor allele of TRIB3 polymorphism for DI and ISI level was estimated by a linear regression analysis. The OR per minor allele of TRIB3 polymorphism for IGR and the OR of DI and ISI for IGR was estimated by logistic regression analysis including age and sex as confounders.

A $p$ value $<0.05$ was considered significant. All analyses were performed using SPSS Version 15.0 (Chicago, IL, USA) and SAS Release 9.1 (SAS Institute, Cary, NC, USA).

\section{Results}

TRIB3 Q84R polymorphism and abnormal glucose homeostasis in samples 1 and 2

For sample 1, the proportion of the TRIB3 genotypes was significantly different in NG participants as compared with individuals with IGR, with the risk of IGR being increased in individuals carrying the R84 variant (Table 1). Similar data were obtained for sample 2 (Table 1). Minimal differences were observed after adjusting also for BMI (OR 1.62, 95\% CI 1.18-2.22 and OR 1.64, 95\% CI 1.062.52 in samples 1 and 2, respectively). When data from the two studies were pooled and analysed the OR and $95 \% \mathrm{CI}$ for IGR was $1.53,1.20-1.95, p=0.0005$. This pooled analysis yielded: for IFG ( $n=131$ case individuals), OR 1.86 and $95 \%$ CI $1.30-2.65$; for IGT $(n=273$ case individuals), OR 1.53 and $95 \%$ CI $1.15-2.03$; and for type 2 diabetes ( $n=63$ case individuals) OR $1.24,95 \%$ CI 0.74 2.08. Although this trend is intriguing and might deserve further attention, the number of individuals in each subgroup is too small to allow any conclusion to be drawn. The results of meta-analysis of these and recently published data, indicating a significant association with abnormalities of glucose homeostasis (either type 2 diabetes or IGR), are shown in Fig. 1a. Given the stronger association with earlyonset type 2 diabetes (i.e. before age 45 years) observed in our previous study [9], meta-analysis was also performed for early-onset forms of abnormal glucose homeostasis (Fig. 1b).

TRIB3 Q84R polymorphism and intermediate metabolic traits in sample 1

Baseline characteristics The baseline features of individuals in sample 1, stratified according to the TRIB3 Q84R genotype, are shown in Table 2 and indicate that the R84 variant, especially in the homozygous state, was associated with higher fasting insulin and HOMA-IR index (Table 2). Also, fasting glucose and triacylglycerol levels tended to be different across genotype groups, though the differences did not reach statistical significance (Table 2).

Glucose and insulin profiles during OGTT Repeatedmeasurements ANCOVA models to assess differences across genotypes over time showed that plasma glucose and insulin levels during OGTT were progressively higher

Table 1 Risk of IGR according to TRIB3 Q84R genotype in two independent samples of Italian whites

\begin{tabular}{|c|c|c|c|c|c|c|}
\hline \multirow[t]{2}{*}{ Sample } & \multirow[t]{2}{*}{$n$} & \multicolumn{3}{|c|}{ TRIB3 Q84R genotype } & \multirow[t]{2}{*}{ OR $(95 \%$ CI $)$} & \multirow[t]{2}{*}{$p$ value } \\
\hline & & QQ $n(\%)$ & $\mathrm{QR} n(\%)$ & $\mathrm{RR} n(\%)$ & & \\
\hline Sample 1 & 791 & & & & & \\
\hline $\begin{array}{l}\text { NG } \\
\text { IGR }\end{array}$ & $\begin{array}{l}465 \\
326\end{array}$ & $\begin{array}{l}352(75.7) \\
213(65.3)\end{array}$ & $\begin{array}{l}108(23.2) \\
104(31.9)\end{array}$ & $\begin{array}{l}5(1.1) \\
9(2.8)\end{array}$ & $1.54(1.15-2.06)$ & 0.004 \\
\hline Sample 2 & 374 & & & & & \\
\hline $\begin{array}{l}\text { NG } \\
\text { IGR }\end{array}$ & $\begin{array}{l}233 \\
141\end{array}$ & $\begin{array}{r}181(77.7) \\
96(68.1)\end{array}$ & $\begin{array}{l}45(19.3) \\
41(29.1)\end{array}$ & $\begin{array}{l}7(3.0) \\
4(2.8)\end{array}$ & $1.63(1.06-2.51)$ & 0.027 \\
\hline
\end{tabular}

Data are adjusted for age and sex

Sample 1: individuals were recruited at the Endocrine Unit of Garibaldi Hospital in Catania, Eastern Sicily, Italy

Sample 2: individuals were recruited at the Department of Clinical Sciences of 'Sapienza', University of Rome, Rome, Italy 
Fig. 1 Individual meta-analysis of six case-control studies. The cumulative effect of four published [9] and the two unpublished present studies on the association between TRIB3 Q84R polymorphism and abnormalities of glucose homeostasis (either type 2 diabetes or IGR) diagnosed either at any age (a) or at age $<45$ years (b) was tested by a fixed-effects model. No heterogeneity was observed across studies ( $p$ values for sample-bygenotype interaction $=0.17$ and 0.36 , respectively). ORs and 95\% CIs for the additive genetic model are shown. The sizes of OR symbols are proportional to the study sample size. The data from all samples were adjusted for age and sex (a) and for sex (b). SGR, San Giovanni

Rotondo, Italy. ${ }^{\mathrm{a} C}$ Cases were patients with frank type 2 diabetes. ${ }^{\mathrm{b}}$ Cases were patients with either impaired fasting glucose and/or impaired glucose tolerance or type 2 diabetes diagnosed at OGTT

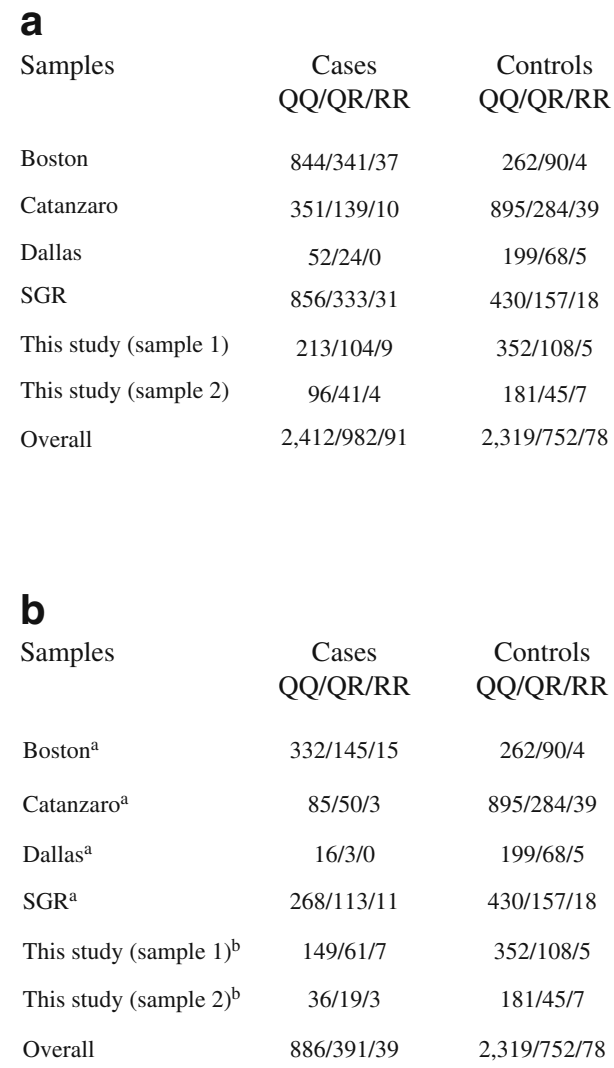

OR (95\% CI), $p$ value

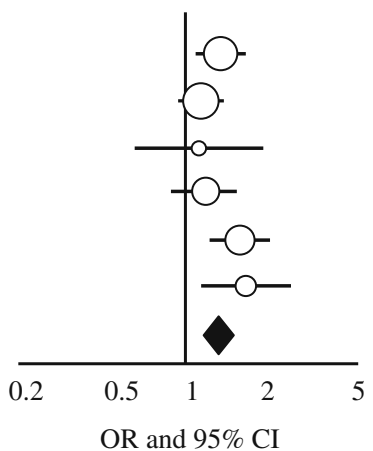

1.28 (1.01-1.64), 0.04 1.06 (0.85-1.32), 0.60 1.04 (0.56-1.92), 0.90 1.09 (0.79-1.49), 0.60

1.54 (1.15-2.06), 0.004 1.63 (1.06-2.51), 0.03 1.19 (1.06-1.34), 0.003 5

OR $(95 \% \mathrm{CI}), p$ value

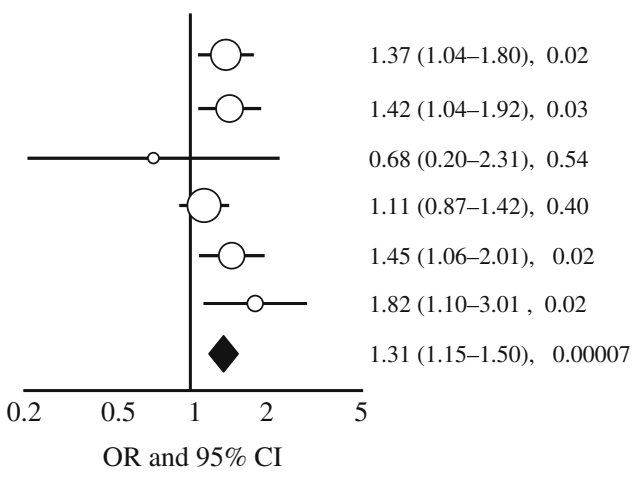

in $\mathrm{QR}$ and $\mathrm{RR}$ individuals as compared with wild-type QQ individuals (age-, sex- and BMI-adjusted $p=0.023$ for glucose and $p=0.009$ for insulin; Fig. 2a, b).

OGTT-derived data on insulin sensitivity, insulin secretion and their interplay ISI was progressively lower in QQ, QR and RR (10.1 $\pm 7.3,9.3 \pm 6.7$ and 5.4 \pm 2.4 , adjusted $p=$ $0.005)$. Absolute values of early-phase insulin secretion, as indicated by the IGI, were not significantly different across
TRIB3 genotypes $(203.7 \pm 135.2,197.0 \pm 165.8$ and $192.0 \pm$ 115.9 in $\mathrm{QQ}, \mathrm{QR}$ and RR individuals; adjusted $p=0.83$ ). Most importantly in the specific context of this study, when early-phase insulin secretion was adjusted for insulin sensitivity, as indicated by the DI, a significant and progressive reduction across the three genotype groups was observed $(2,090.6 \pm 2,311.1,1,774.7 \pm 2,092.4$ and $1,055.2 \pm 718.4$ in QQ, QR and RR, respectively, adjusted $p=0.043$ ). Meta-analysis was then performed on data on DI
Table 2 Anthropometric and biochemical characteristics of the study participants in sample 1 according to TRIB3 Q84R genotype

Data are means \pm SD or percentage of total

${ }^{\text {a }}$ Adjusted for age, sex and BMI

\begin{tabular}{lllll}
\hline Characteristic & \multicolumn{2}{l}{ TRIB3 Q84R genotype } & \\
\cline { 2 - 5 } & QQ & QR & RR & $p$ value \\
\hline$n$ (men/women) & $565(166 / 399)$ & $212(68 / 144)$ & $14(4 / 10)$ & 0.55 \\
Age (years) & $34.9 \pm 12.0$ & $37.6 \pm 11.3$ & $35.1 \pm 12.5$ & 0.013 \\
BMI (kg/m $\left.{ }^{2}\right)$ & $38.6 \pm 10.8$ & $38.8 \pm 9.9$ & $39.6 \pm 7.8$ & 0.69 \\
Fasting glucose (mmol/l) & $5.2 \pm 0.7$ & $5.3 \pm 0.7$ & $5.4 \pm 0.9$ & $0.10^{\mathrm{a}}$ \\
Fasting insulin (pmol/l) & $111.4 \pm 66.5$ & $114.0 \pm 67.7$ & $150.6 \pm 51.9$ & $0.020^{\mathrm{a}}$ \\
HOMA-IR & $3.8 \pm 2.5$ & $4.0 \pm 2.6$ & $5.3 \pm 2.4$ & $0.012^{\mathrm{a}}$ \\
Triacylglycerol (mmol/1) & $1.3 \pm 0.8$ & $1.4 \pm 0.7$ & $1.5 \pm 0.8$ & $0.081^{\mathrm{a}}$ \\
HDL-cholesterol (mmol/1) & $1.2 \pm 0.3$ & $1.1 \pm 0.3$ & $1.2 \pm 0.4$ & $0.97^{\mathrm{a}}$ \\
Metabolic syndrome (\%) & $239(42.3 \%)$ & $98(46.2 \%)$ & $8(57.1 \%)$ & $0.42^{\mathrm{a}}$ \\
\hline
\end{tabular}


a

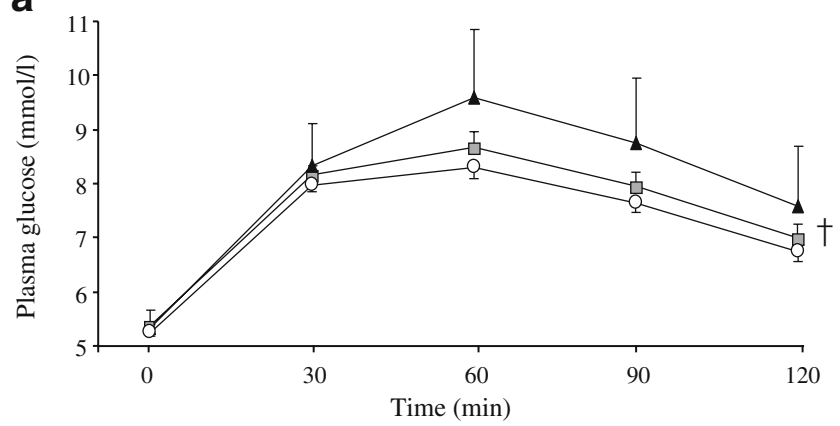

b

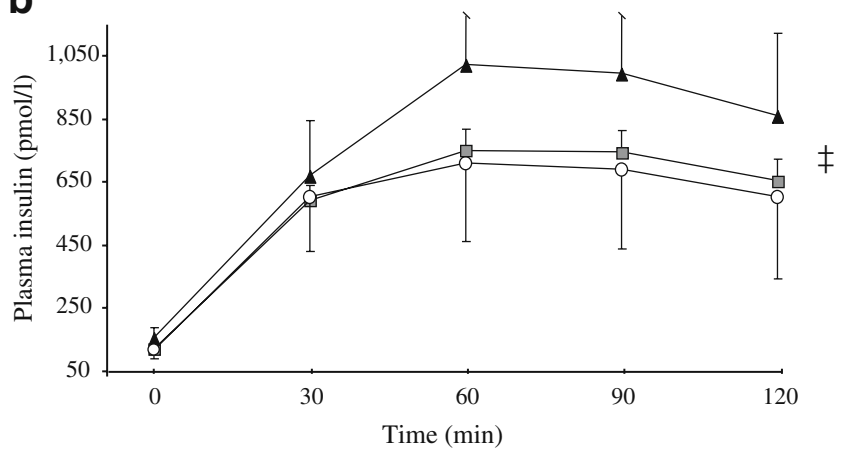

Fig. 2 Glucose (a) and insulin (b) levels during OGTT in QQ (white circles), QR (grey squares), and RR (black triangles) individuals from sample $1 ;{ }^{\dagger} p=0.023 ;{ }^{\star} p=0.009$. Repeated-measurements ANCOVA models were used to assess differences across genotypes over time according to an additive genetic model. All data were adjusted for sex, age and BMI

from this study and recently published data reporting a similar association [9]; the results obtained in a total number of 1,436 individuals, confirmed the association (adjusted $\beta$ (SE) of $\log$ DI for each R84 allele $=-0.15$ $(0.04)$, adjusted $p=0.0007)$. We then asked whether the R84 effect on the risk of IGR was mediated either by DI or by ISI changes and tested it by a 'triangulation approach' in sample 1, for whom all the data needed to test these hypotheses were available. The observed OR for IGR (1.54) was virtually identical to the expected one when DI (i.e. 1.51; Fig. 3) was taken into account, but not when ISI (i.e. 1.25) was taken into account, thus supporting the idea that changes in DI, more than in ISI, are likely to mediate the effect of R84 on the risk of IGR.

TRIB3 Q84R polymorphism and insulin sensitivity in sample $3 \mathrm{In}$ order to get further insights about the role of the R84 variant on insulin sensitivity, an additional sample of 394 individuals who underwent the euglycaemichyperinsulinaemic glucose clamp was analysed. Preliminary data on 178 of these individuals have been already reported [6]. Insulin sensitivity as indicated by insulin-stimulated glucose disposal was progressively lower in QQ, QR and RR individuals $(38.8 \pm 17.7,33.8 \pm 14.4$ and $31.6 \pm 13.3 \mu \mathrm{mol}$ $\min ^{-1} \mathrm{~kg}^{-1}$, respectively, $p=0.022$ ). This association did not change much after adjusting for age, sex and BMI $(p=0.05)$.

\section{Discussion}

We here report that the TRIB3 $\mathrm{R} 84$ variant is associated with altered glucose homeostasis in Europeans from Italy, recruited on the basis of having a fasting glucose level in the non-diabetic range (i.e. below $7.0 \mathrm{mmol} / \mathrm{l}$ ). Virtually identical results were obtained in two different heterogeneous samples, thus providing evidence of formal replication. Of note, obtaining similar results in samples which are clinically heterogeneous increases the chance that our finding is a generalisable one.

When the current data are meta-analysed with those previously published for the association with type 2 diabetes [9], the statistical association becomes quite robust, especially for abnormal glucose homeostasis before age 45 years. In addition, we also report that individuals carrying the R84 variant are characterised by altered interplay between insulin sensitivity and secretion as indicated by reduced DI values for OGTT. These data too represent a formal replication of a previous finding [9]; also in this case, when present and previous [9] data are metaanalysed a quite robustly significant association is reached. Taken together, these and previous [9] data suggest that the TRIB3 R84 variant exerts a deleterious role on glucose homeostasis by affecting the interplay between insulin sensitivity and insulin secretion; the subtle equilibrium is instrumental for the maintenance of glucose homeostasis

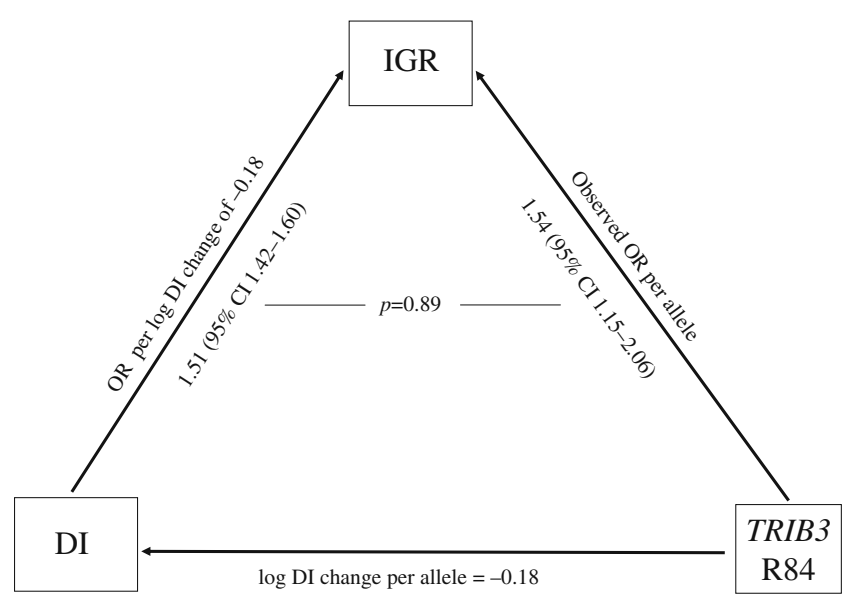

Fig. 3 Use of a 'triangulation approach' in sample 1. Associations between SNP Q84R and both DI (delta of log DI values per allele) and IGR ('observed OR' per allele), and between DI and IGR. The latter association is used to assume 'expected OR' between Q84R and IGR, which is then compared with the 'observed OR'. All data are given according to additive genetic model (i.e. the effect of each at risk R84 allele) 
$[22,23]$. To test this possibility a 'triangulation approach' was used [12]; as a matter of fact, the observed and expected ORs for IGR were virtually identical, strongly supporting our hypothesis.

A few differences between present and previous [9] findings on insulin sensitivity and the risk of IGR as well as the absolute amount of early phase insulin secretion deserve some comment. In contrast to the present study, no association between the R84 variant and insulin resistance and IGR was previously observed [9]. It is of note that, as per different selection criteria, individuals with type 2 diabetes at OGTT were included in the present but not in the previous [9] study, thus limiting in the latter one the distribution toward the high end of the spectrum of insulin sensitivity. This might have reduced the power to detect a significant association between the R84 variant and both insulin resistance and abnormalities of glucose homeostasis. Overall, given these conflicting results we tested in an additional sample the association of the R84 variant with insulin resistance as measured by the gold-standard technique, the euglycaemic-hyperinsulinaemic glucose clamp [13]. The data obtained further support the role of R84 in affecting insulin sensitivity.

A second difference refers to absolute values of earlyphase insulin secretion, as indicated by the IGI, which was reduced in R84 carriers in a previous [9], but not in the present, study. Absolute insulin secretion is partly determined by insulin sensitivity in the sense that it depends on the amount of insulin needed to maintain normoglycaemia. It is therefore expected that a condition of insulin resistance, as in R84 carriers in the present study, exerts an increased stimulus for insulin secretion; thus, even in the presence of beta cell dysfunction, insulin resistance may well explain similar IGI values observed across genotype groups as was found in the present study. What is really of note from a pathophysiological perspective is that, regardless of the observed differences in absolute values of insulin secretion and/or sensitivity, the interplay between these two variables, as indicated by DI-the best predictor of future type 2 diabetes [22, 23] - is similarly reduced in R84 carriers both in the previous [9] and the present study. As for any genetic study of complex traits, data replication is of particular importance in order to reduce the risk of falsepositive results and to accumulate data aimed at establishing a new finding by reaching a high level of statistical significance.

In functional studies on transfected cells, an inhibitory role of R84 variant on insulin-stimulated Akt phosphorylation has been observed in vitro in both cells from peripheral insulin target tissues $[6,7]$ and insulin-secreting beta cells (C. Wee Liew, J. Bochenski, J. Hu, A.S. Krowleski and R.N. Kulkarni, unpublished data), making plausible a direct role of this variant on both in vivo insulin sensitivity and insulin secretion. A similar deleterious role on both insulin sensitivity and secretion has been reported for other nonsynonymous genetic variations of insulin signalling genes, including IRS-I (also known as IRSI) G972R [24-26] and ENPP1 K121Q [27-29] polymorphisms. Taken together, present and previous genetic data are consistent with a pathogenic role of altered insulin signalling in inducing both peripheral insulin resistance and impaired insulin secretion [30].

In conclusion, the present study serves the important function of confirming that, among individuals of Southern European ancestry, the TRIB3 R84 variant is associated with an increased risk of abnormal glucose homeostasis. The present data also strengthen the previous hypothesis [9] that this association is mediated by an abnormal interplay between insulin sensitivity and insulin secretion; despite this replication we acknowledge that, as in any crosssectional study, association cannot guarantee a cause-andeffect relationship and that only prospective data can establish such a relationship. We also acknowledge that further replication attempts - either by studying new samples (possibly comprising individuals other than Southern Europeans) or by in silico analysis of databases that are presently not publicly available - are needed before TRIB3 can be conclusively listed among genes able to modulate the susceptibility to abnormal glucose homeostasis.

Acknowledgements This work was partly supported by Grants from: the Italian Ministry of Health (Ricerca Finalizzata 2006 to S. Prudente and F. Barbetti, Ricerca Corrente 2008 and 2009 to S. Prudente and V. Trischitta), the Italian Ministry of University and Research (PRIN 2005 to L. Frittitta and PRIN 2005 and 2007 to V. Trischitta), the European Community (FP6 EUGENE2 no. LSHM-CT2004-512013 Grant to G. Sesti) and University of Catania (2005 and 2006 to L. Frittitta).

We thank N. Abate (Division of Endocrinology and Metabolism, University of Texas Medical Branch, Galveston, TX, USA) and A. Doria (Research Division, Joslin Diabetes Center, Boston, MA, USA) for allowing us to meta-analyse our present data with individual data from the GENIUS consortium's case-control study for type 2 diabetes, the results of which have been previously published [9].

Duality of interest The authors declare that there is no duality of interest associated with this manuscript.

\section{References}

1. Reaven GM, Banting Lecture (1988) Role of insulin resistance in human disease. Diabetes 37:1595-1607

2. Kahn CR, Banting Lecture (1994) Insulin action, diabetogenes, and the cause of type II diabetes. Diabetes 43:1066-1084

3. Martin BC, Warram JH, Krolewski AS, Bergman RN, Soeldner JS, Kahn CR (1992) Role of glucose and insulin resistance in development of type 2 diabetes mellitus: results of a 25 -year follow-up study. Lancet 340:925-929

4. Weyer C, Bogardus C, Mott DM, Pratley RE (1999) The natural history of insulin secretory dysfunction and insulin resistance in 
the pathogenesis of type 2 diabetes mellitus. J Clin Invest 104:787-794

5. Du K, Herzig S, Kulkarni RN, Montminy M (2003) TRB3: a tribbles homolog that inhibits Akt/PKB activation by insulin in liver. Science 6:1574-1577

6. Prudente S, Hribal ML, Flex E et al (2005) The functional Q84R polymorphism of mammalian Tribbles homolog TRB3 is associated with insulin resistance and related cardiovascular risk in Caucasians from Italy. Diabetes 54:2807-2811

7. Andreozzi F, Formoso G, Prudente S et al (2008) TRIB3 R84 variant is associated with impaired insulin mediated nitric oxide production in human endothelial cells. Arterioscler Thromb Vasc Biol 28:1355-1360

8. Gong HP, Wang ZH, Jiang H et al (2009) TRIB3 functional Q84R polymorphism is a risk factor for metabolic syndrome and carotid atherosclerosis. Diabetes Care 32:1311-1313

9. Prudente S, Scarpelli D, Chandalia M et al (2009) The TRIB3 Q84R polymorphism and risk of early-onset type 2 diabetes. $\mathrm{J}$ Clin Endocrinol Metab 94:190-196

10. Zeggini E, Scott LJ, Saxena R et al (2008) Meta-analysis of genome-wide association data and large-scale replication identifies additional susceptibility loci for type 2 diabetes. Nat Genet 40:638-645

11. Diabetes Genetics Initiative of Broad Institute of Harvard and MIT, Lund University, and Novartis Institutes of BioMedical Research, Saxena R, Voight BF, Lyssenko V et al (2007) Genomewide association analysis identifies loci for type 2 diabetes and triglyceride levels. Science 316:1331-1336

12. Freathy RM, Timpson NJ, Lawlor DA et al (2008) Common variation in the FTO gene alters diabetes-related metabolic traits to the extent expected given its effect on BMI. Diabetes 57:1419-1426

13. DeFronzo RA, Tobin JD, Andres R (1979) Glucose clamp technique: a method for quantifying insulin secretion and resistance. Am J Physiol 237:E214-223

14. Matthews DR, Hosker JP, Rudenski AS, Naylor BA, Treacher DF, Turner RC (1985) Homeostasis model assessment: insulin resistance and beta-cell function from fasting plasma glucose and insulin concentrations in man. Diabetologia 28:412-419

15. Singer JD, Willett JB (2003) Applied longitudinal data analysis: modeling change and event occurrence. Oxford University Press, New York

16. Wigginton JE, Cutler DJ, Abecasis GR (2005) A note on exact tests of Hardy-Weinberg equilibrium. Am J Hum Gen 76:887-893
17. Ioannidis JP, Rosenberg PS, Goedert JJ, O'Brien TR (2002) International meta-analysis of HIV host genetics. Commentary: meta-analysis of individual participants' data in genetic epidemiology. Am J Epidemiol 156:204-210

18. Higgins JP, Whitehead A, Turner RM, Omar RZ, Thompson SG (2001) Meta analysis of continuous outcome data from individual patients. Stat Med 20:2219-2241

19. Sutton AJ, Kendrick D, Coupland CA (2008) Meta-analysis of individual- and aggregate-level data. Stat Med 27:651-669

20. Begg CB, Mazumdar M (1994) Operating characteristics of a rank correlation test for publication bias. Biometrics 50:1088-1101

21. Egger M, Davey Smith G, Schnedier M, Minder C (1997) Bias in meta-analysis detected by a simple graphical test. BMJ 315:629634

22. Lyssenko V, Almgren P, Anevski D et al (2005) Predictors of and longitudinal changes in insulin sensitivity and secretion preceding onset of type 2 diabetes. Diabetes 54:166-174

23. Abdul-Ghani MA, Williams K, DeFronzo RA, Stern M (2007) What is the best predictor of future type 2 diabetes? Diabetes Care 30:1544-1548

24. Clausen JO, Hansen T, Bjorbaek C et al (1995) Insulin resistance: interactions between obesity and a common variant of insulin receptor substrate-1. Lancet 346:397-402

25. Koch M, Machicao F, Haring H (2001) The Gly972Arg polymorphism in the insulin receptor substrate-1 gene contributes to the variation in insulin secretion in normal glucose-tolerant humans. Diabetes 50:882-885

26. Marchetti P, Lupi R, Federici M et al (2002) Insulin secretory function is impaired in isolated human islets carrying the Gly $(972) \rightarrow$ Arg IRS-1 polymorphism. Diabetes 51:1419-1424

27. Pizzuti A, Frittitta L, Argiolas A et al (1999) Polymorphism (K121Q) of the human glycoprotein PC-1 gene coding region is strongly associated with insulin resistance. Diabetes 48:18811884

28. McAteer JB, Prudente S, Bacci S et al (2008) The ENPP1 K121Q polymorphism is associated with type 2 diabetes in European populations: evidence from an updated meta-analysis in 42,042 subjects. Diabetes 4:1125-1130

29. Baratta R, Rossetti P, Prudente S et al (2008) Role of the ENPP1 K121Q polymorphism on glucose homeostasis. Diabetes 57: 3360-3364

30. Biddinger SB, Kahn CR (2006) From mice to men: insights into the insulin resistance syndromes. Annu Rev Physiol 68:123-158 